\title{
Endoscopic introduction of an over-the-scope clip through an overtube to close a gastrocutaneous fistula in a patient with a complex upper esophageal stenosis
}
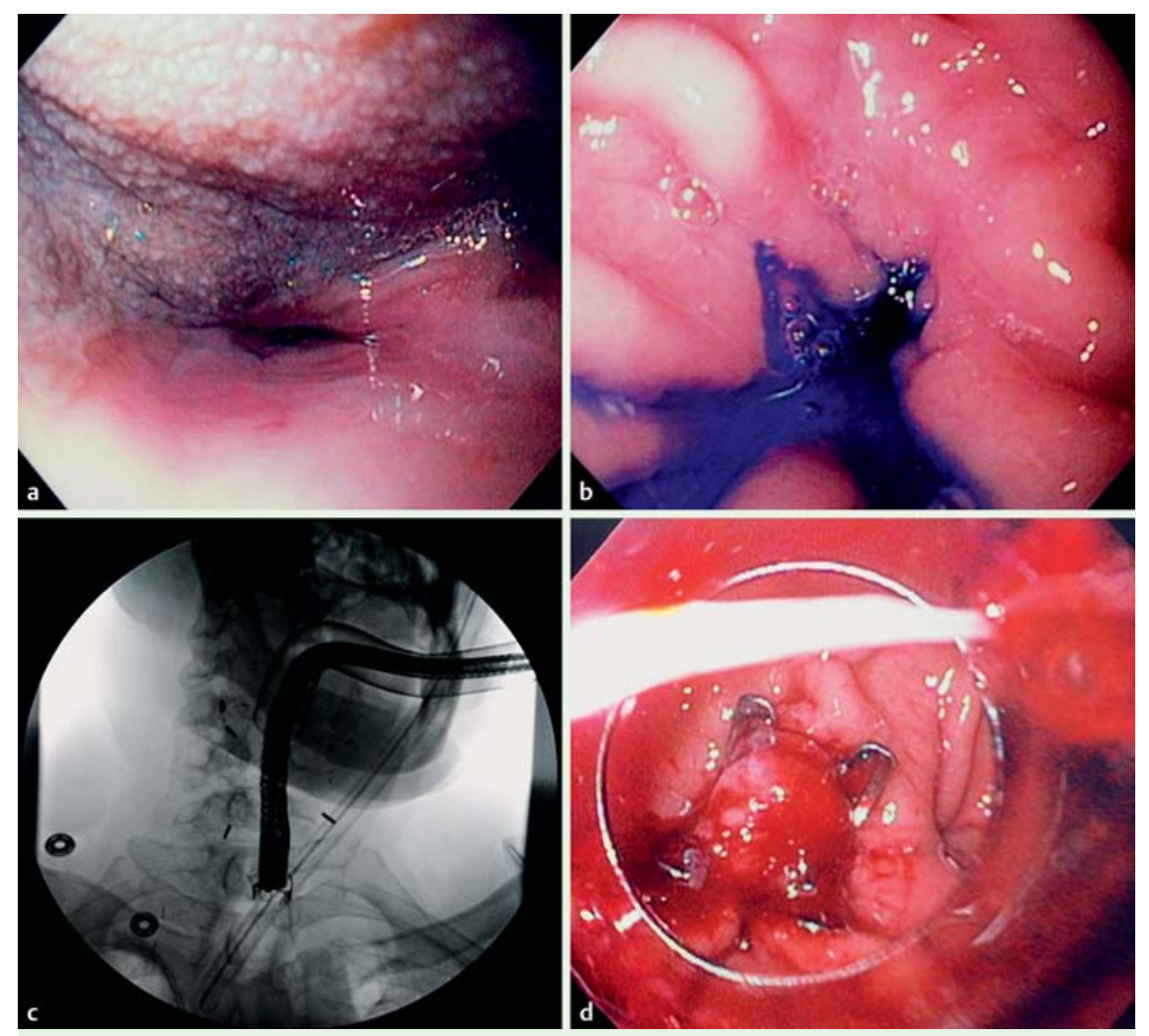

Fig. 1 a Gastrocutaneous fistula in a 51-year-old man, which developed after the removal of a gastrostomy tube. $\mathbf{b}$ The fistula was found between the gastric folds by injecting indigo carmine through the opening in the skin. $\mathbf{c}$ The presence of an overtube allowed passage of the scope loaded with the over-the-scope clip (OTSC). The overtube functioned as a giant working channel. $\mathbf{d}$ The OTSC was successfully deployed over the fistula, which closed immediately.

A 51-year-old man with a history of laryngeal cancer requiring total laryngectomy and placement of a forearm skin graft into the hypopharynx developed a nonhealing gastrocutaneous fistula after removal of a gastrostomy tube ( Fig.1a). The injection of indigo carmine through the skin made it possible to determine the precise location of the 3- to 4-mm-diameter gastrocutaneous fistula within thickened gastric folds ( $\nabla$ Fig. 1 b). A gastroscope could be passed into the stomach after an esophageal stenosis between the hypopharynx and upper esophageal sphincter, induced by radiation fibrosis and the presence of a forearm skin graft, had been dilated sequentially with 9-, 10-, and 12-mm Savary bougies; however, it was still not possible to pass the fistula-closing device, an overthe-scope clip (OTSC System; Ovesco En- doscopy, Tübingen, Germany), through the hypopharynx.

Contrast esophagography after the dilation procedure did not show any endoluminal esophageal damage, so an overtube (Guardus overtube-esophageal; inner diameter $16.7 \mathrm{~mm}$, outer diameter $19.9 \mathrm{~mm}$; US Endoscopy, Mentor, Ohio, USA) was inserted into the esophagus. The large-diameter overtube served as a "giant working channel," permitting passage of the endoscope loaded with the 11/6t OTSC device ( Fig. 1 c). Therefore, the overtube was used mainly to open the space to facilitate passage of the scope loaded with the device, especially during endotracheal intubation (this procedure was performed with the patient under general anesthesia).
The fistula was closed successfully ( $\nabla$ Fig.1d). Closure was documented by administering water-soluble contrast through the endoscope. The overtube and endoscope were removed, and the additional administration of contrast at the hypopharynx and upper esophagus did not reveal any damage, leak, or extravasation.

This case is an example of "extreme endoscopy." First, the complex fistula could be found by using interventional chromoendoscopy. The esophageal stricture was dilated, and an overtube was inserted into the stomach. The overtube served as a bridge of the upper esophageal stenosis, allowing smooth passage of the scope with a large fistula-closing device, such as the OTSC System.

In summary, an impossible situation was converted into a therapeutic solution by combining the use of techniques and equipment widely available in the endoscopy suite, such as fluoroscopy, overtubes, dilation balloons, clipping devices, and chromoendoscopy.

\section{Endoscopy_UCTN_Code_TTT_1AO_2AI}

\section{Competing interests: None}

\section{Marco Aurélio D’Assuncao, Paul T. Kröner, Sandhya Mudumbi, Klaus Mönkemüller}

Basil I. Hirschowitz Endoscopic Center of Excellence, Division of Gastroenterology and Hepatology, University of Alabama at Birmingham, Alabama, USA

\section{Bibliography}

DOI http://dx.doi.org/

10.1055/s-0034-1392561

Endoscopy 2015; 47: E412

(c) Georg Thieme Verlag KG

Stuttgart · New York

ISSN 0013-726X

\section{Corresponding author}

\section{Klaus Mönkemüller, MD, PhD, FASGE}

Division of Gastroenterology and Hepatology

Basil I. Hirschowitz Endoscopic

Center of Excellence

Endoscopy Unit, JT 664

619 19th Street $S$

Birmingham, AL 35249

USA

Fax: +1-205-297-9411

klaus1@uab.edu 